\title{
Implementación y certificación de MoProSoft en una pequeña empresa desarrolladora de software: lecciones aprendidas de cuatro iteraciones de mejora
}

\author{
Patricia Morillo $^{1}$, Marco Vizcardo ${ }^{1}$, Víctor Sanchez ${ }^{1}$, Abraham Dávila ${ }^{2}$ \\ ${ }^{1}$ Facultad de Ciencias e Ingeniería - Pontificia Universidad Católica del Perú (PUCP) \\ Av. Universitaria 1801, San Miguel, Lima 32, - Perú \\ ${ }^{2}$ Departmento de Ingeniería - Pontificia Universidad Católica del Perú \\ Av. Universitaria 1801, San Miguel, Lima 32, Perú. \\ \{pmorillo, mvizcardo, victor.sanchezc, abraham.davila\}@ pucp.edu.pe

\begin{abstract}
Resumen. La mejora de proceso de una pequeña empresa que desarrolla software presenta algunas características particulares propias a su tamaño y que requieren ser resueltas de acuerdo a la realidad de la propia empresa. En este artículo se presenta las lecciones aprendidas en tres ciclos de mejora de procesos y uno de preparación para la certificación de madurez de una pequeña empresa que desarrolla software utilizando MoProSoft dentro de un programa de mejora de procesos dirigido desde el entorno académico.
\end{abstract}

\begin{abstract}
The process improvement in a software development small company has special characteristics relative to their size and need to be solved according to the company reality. This article presents lessons learned from three cycles of process improvement and one cycle of preparation to get the organizational maturity certification of a small company that develops software using MoProSoft within a program of process improvement led from the academic environment.
\end{abstract}

\section{Introducción}

La productividad y la calidad son temas que preocupan a las empresas informáticas por la presión que cada vez más tienen del mundo globalizado y que inevitablemente afecta su negocio. La baja productividad conlleva a perder dinero y la baja la calidad del producto conlleva, por lo general, a perder clientes; sin embargo, ambos están muy influenciados por la calidad de los procesos.

En este contexto se han desarrollado distintos modelos de calidad de procesos como CMMI o ISO/IEC 12207 con ISO/IEC 15504, que tienen aceptación internacional. Sin embargo no han sido del todo acogidos por las pequeñas y medianas empresas (PYME) desarrolladoras de software [Oktaba 2006], [Richardson 2007], [Jones 2005], [Gresse 2006], [Allen 2003]; por lo que se han propuesto modelos para pequeñas empresas como es el caso de MoProSoft en México [Oktaba et al. 2005], MR.MPS en Brasil [Weber, 2004] y SIMEC-SW en Colombia [Hurtado 2005].

Considerando el contexto y la necesidad de las pymes desarrolladoras de software, se constituye en el 2006, el proyecto COMPETISOFT, que se propone como 
una alternativa conjunta en la región Iberoamericana [COMPETISOFT, 2008]. Dentro de este Proyecto, el componente Peruano de la PUCP (Pontificia Universidad Católica del Perú) desarrolló pruebas piloto con varias pymes y además extendió el proyecto a dos fases siguientes para concretar logros para el Perú.

Este artículo presenta la experiencia de implantación de MoProSoft en una pequeña empresa desarrolladora de software a lo largo de tres ciclos de mejora dirigidos desde el ámbito académico y un ciclo de preparación para la certificación; dentro del marco de un proyecto mayor donde participaron otras 22 empresas en distintas fases. El artículo se organiza de la siguiente manera: la sección 2 presenta brevemente los modelos de utilizados en el Proyecto, la sección 3 presenta una descripción de la Empresa en estudio, la sección 4 el trabajo desarrollado en cada ciclo de mejora y los resultados obtenidos; y la sección 5, la discusión final de la experiencia.

\section{Proyecto COMPETISOFT - Componente peruano}

COMPETISOFT es la denominación de un proyecto que para nuestro caso (Perú) se concretó a través de tres fases consecutivas, utilizando modelos de calidad y diferentes alcances y propósitos alineados en el tiempo. El nombre COMPETISOFT corresponde originalmente a un proyecto Ibero-Americano en la que el equipo de la PUCP participó y sobre esa experiencia se extiendió a dos proyectos adicionales (que denominaremos fases) para dar una idea de continuidad de conceptos y modelos a las empresas, profesionales y al país. A continuación se describe las fases y modelos del Proyecto.

\subsection{Fases del proyecto COMPETISOFT para Perú}

La primera fase fue el proyecto COMPETISOFT financiado por el CYTED que se desarrolló entre el 2006 y el 2008 [Competisoft 2008]. COMPETISOFT fue un esfuerzo conjunto e iberoamericano de investigadores y profesionales interesados en incrementar la competitividad de la industria de software en la región [Oktaba 2007]. Durante esta Fase se desarrollaron, entre otros, proyectos de mejora en algunas pymes que desarrollan software a manera de pruebas controladas de los modelos que se trabajaron en COMPETISOFT. Dependiendo de la fecha de incorporación de las empresas algunas completaron uno y otras dos ciclos de mejora; durante el último año de la Fase 1.

La segunda fase fue un proyecto de replicación de conocimientos y experiencias (adquiridas en la primera fase) a otras ciudades (Arequipa y Trujillo además de Lima), otras universidades y empresas (algunas de la Fase 1 continuaron); este proyecto se desarrolló durante el 2009 y fue financiado por un fondo de la PUCP y empresas.

La tercera fase fue un proyecto para el establecimiento formal y basado en estándares internacionales de una certificación para pymes desarrolladoras de software utilizando los modelos del Proyecto; esta Fase se realizó entre el 2010 y el primer semestre del 2011 con financiamiento de FINCYT y de la empresa ACKLIS SAC. En esta Fase participaron empresas seleccionadas en un ciclo de mejora para prepararse y lograr la certificación bajo este nuevo esquema basado en normas internacionales.

\subsection{Esquema de trabajo con empresas y estudiantes}

Para desarrollar un esquema adecuado de trabajo, con las empresas y los estudiantes en lo que respecta a la mejora de procesos, se concretó algunas actividades previamente. 
Para el caso de las empresas, se realizó: la convocatoria (en cada fase), charlas de sensibilización colectiva e individual, talleres sobre los modelos y temas generales de ingeniería de software. En algunos casos, principalmente en la Fase 1, se firmaron acuerdos de colaboración entre la empresa y la PUCP. Para el caso de los estudiantes se realizó: la convocatoria (en cada fase), talleres sobre los modelos y como aplicarlos en las pymes, reuniones de trabajo conjunto para monitoreo de los proyectos y participación en la revisión y modificación de documentos para cada ciclo de mejora.

El esquema para todos los ciclos de mejora fue que un estudiante era asignado a una empresa donde llevaría adelante un ciclo de mejora. Durante la primera semana se realizaba la inducción al estudiante y se realizaba la evaluación de los procesos de la empresa respecto de MoProSoft con participación del investigador principal; la idea fue que los estudiantes tuviesen un buen conocimiento de la situación actual de la empresa. Luego de la evaluación se formulaba el plan de mejora del ciclo con la participación de la alta dirección, el responsable del proyecto por el lado de la empresa, el estudiante y el investigador principal. La implementación recayó en el estudiante y el responsable del proyecto y los distintos dueños de procesos considerados en la mejora. Al culminar el ciclo de mejora, el investigador principal, realizaba la evaluación final, con participación del estudiante en toda la evaluación pero como parte de la empresa evaluada. Concluido el ciclo de mejora, se acordaba con la empresa si ésta continuaba o no; de continuar se cambiaba al estudiante para el nuevo ciclo de mejora. Cada ciclo de mejora duró entre 5 y 6 meses. Para lograr un buen desempeño los estudiantes fueron constantemente monitoreados por un investigador asignado y además tuvieron la responsabilidad de ser el canal de comunicación entre el equipo del Proyecto y la empresa participante.

\subsection{Modelo de Procesos}

Para el proyecto COMPETISOFT (Fase 1) se adoptó trabajar con MoProSoft como modelo base para definir un modelo a partir de las experiencias del proyecto, dicho nuevo modelo se denominó COMPETISOFT [Competisoft 2008]; sin embargo, en esencia son muy parecidos. MoProSoft es el modelo de procesos desarrollado para la industria de software mexicana [Oktaba et al. 2005] y tiene nueve procesos organizados por categorías (ver Figura 1). MoProSoft fue adoptado como base para el nuevo estándar internacional ISO/IEC 29110 en el 2007 [Laporte et al. 2008] y sobre esa base se decidió trabajar en el Perú a MoProSoft como Norma Técnica Peruana (NTP) y fue publicada como NTP 291.100 en 2009 [NTP 2009]. Todo este esfuerzo, en el Perú, con el interés de posteriormente migrar la NTP 291.100 a la nueva ISO/IEC 29110 de reciente publicación [ISO/IEC 2011] al estar ambas basadas en MoProSoft.

\subsection{Modelo de Mejora}

El modelo de mejora de procesos se denominó PMCompetiSoft y está basado en AgilSPI que es un marco de mejora creado para la industria del software de Colombia [Hurtado, 2005]. PMCompetiSoft está compuesto por 5 macro-actividades: (i) instalación del Programa, se crea una propuesta de mejora; (ii) diagnóstico, se determina cuál es el estado general de los procesos; (iii) formulación, se prioriza y se planifica las iteraciones; (iv) mejora, se ejecuta y se gestiona la mejora; y (v) revisión del programa, se realiza una retroalimentación antes de comenzar la siguiente iteración. 


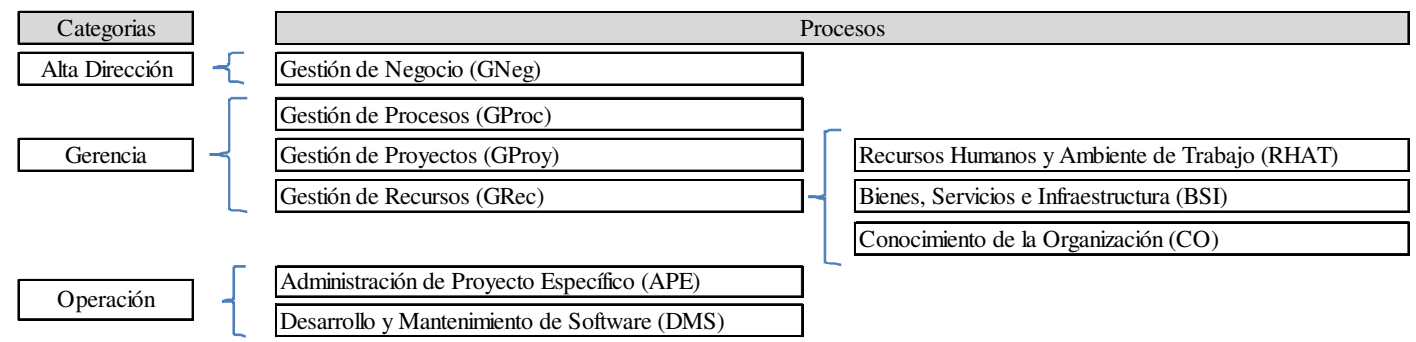

Figura 1. Categoría de Procesos de MoProSoft

\subsection{Modelo de Evaluación}

Para el proyecto COMPETISOFT, Fase 1, se propuso EvalProSoft como modelo de evaluación de MoProSoft. EvalProSoft aplica a las organizaciones dedicadas al desarrollo y/o mantenimiento de software, en particular a aquellas que han utilizado como modelo a MoProSoft para sus procesos [Oktaba et al. 2004]. La capacidad del proceso se evalúa en una escala de 0 a 5 , el valor 0 se asocia al nivel de capacidad más bajo, que significa que no se alcanzó el propósito del proceso de mejora y el valor 5 se asocia al nivel de capacidad más alto y significa que se lograron las metas establecidas; niveles de capacidad basado en ISO/IEC 15504 [ISO 2003], [Pino 2006].

De la revisión por un equipo de evaluadores en ISO/IEC 15504, se determinó que EvalProSoft a pesar de estar basado ISO/IEC 15504, no cumplía con todos los requisitos de la ISO/IEC 15504-2; por lo cual fue necesario desarrollar ampliaciones de modo que sí cumpla con los requisitos de la Norma. Estas ampliaciones permitió el desarrollo de instrumentos para realizar evaluaciones de diferente nivel de rigurosidad (diagnóstica, intermedia y de certificación). La ISO/IEC 15504-2 define un proceso de evaluación y un marco de referencia para las mediciones de los atributos de proceso. En este marco de medición los niveles de calificación utilizados son [ISO 2003]: F de completamente logrado (Fully achieved) que representa entre 85 y $100 \%$, L de ampliamente logrado (Largely achieved) que representa entre 50 y $85 \%, \mathrm{P}$ de parcialmente logrado (Partially achieved) que representa entre 15 y $50 \%$ y $\mathrm{N}$ de no logrado (Not achieved) que representa entre 0 y $15 \%$.

\section{Empresa Alfa}

Para efectos de mantener la confidencialidad de la empresa participante, esta será referida como Alfa. La empresa Alfa es una organización cuyo objetivo es brindar soluciones informáticas orientadas al sector de telecomunicaciones y es el resultado del MBO (Management Buy Out) de la filial en Perú de una empresa informática multinacional cuya presencia en el país se dio desde el año 1997. Alfa se conformó a partir de dicha adquisición en el año 2005 teniendo su sede principal ubicada en LimaPerú y una oficina en otro país de la región. Alfa heredo algunas buenas prácticas de la multinacional, sin embargo, estas prácticas no eran seguidas por resultar difíciles de aplicar ya que no se encontraban adaptadas a la realidad y tamaño de Alfa.

Alfa tiene un fuerte énfasis en el tema de ventas de proyectos, debido a la formación comercial que tiene el gerente general, por tal razón se maneja una gestión de ventas con 3 personas dedicadas a la búsqueda de oportunidades y una gestión de 
proyecto para que gestione todos los proyecto. Alfa cuenta con distintas unidades de negocio como Software de Telecomunicaciones, Infraestructura de Telecomunicaciones, Software Factory, Outsourcing y Mobile Business, Aseguramiento de Calidad, Tecnologías de la Información y PBX, e Investigación, Desarrollo y Capacitación. También cuenta con un área comercial para la comunicación especializada con el cliente.

\section{$4 \quad$ Proceso de Mejora y Resultados}

El proceso de mejora en Alfa se inició en May-2007 ejecutándose dos ciclos de mejora consecutivos que concluyó en Jun-2008, teniendo en promedio cada ciclo 6 meses. El primer ciclo de mejora estuvo a cargo de Patricia Morillo, el segundo ciclo de mejora a cargo de Marco Vizcardo. El tercer ciclo de mejora comenzó en Sep-2009 y culminó en Feb-2010 estando a cargo de Víctor Sánchez. El ciclo de preparación para la certificación se realizó hasta Ago-2010 cuando fue evaluada para la certificación, obteniéndose la misma en Oct-2010; este último esfuerzo contó con la participación de Víctor Sánchez ya como empleado de Alfa.

La Tabla 1 presenta las calificaciones obtenidas por la Alfa en los distintos ciclos de mejora (inicio y fin) y la del proceso de certificación; considerando además que en un periodo no se realizó ciclo de mejora. Se presenta para cada evaluación realizada en el proyecto el grado de adhesión (en porcentaje) y la correspondiente calificación según la ISO/IEC 15504 (indicado en la sección 2.5). Para la adhesión se optó por utilizar una escala valorada entre 1 y 100 , para mostrar de mejor manera el nivel de adhesión de los procesos de Alfa al MoProSoft. A lo largo del proyecto se prefirió usar este esquema de puntuación con números (menos fiable) en lugar de usar las letras (más fiables); pero que permitió conocer mejor su estado de progreso. Además, para los ciclos 1 y 2 se presentan resultados de evaluaciones del nivel de capacidad 1 y para el ciclo 3 se presentan para el nivel de capacidad 1 y 2 .

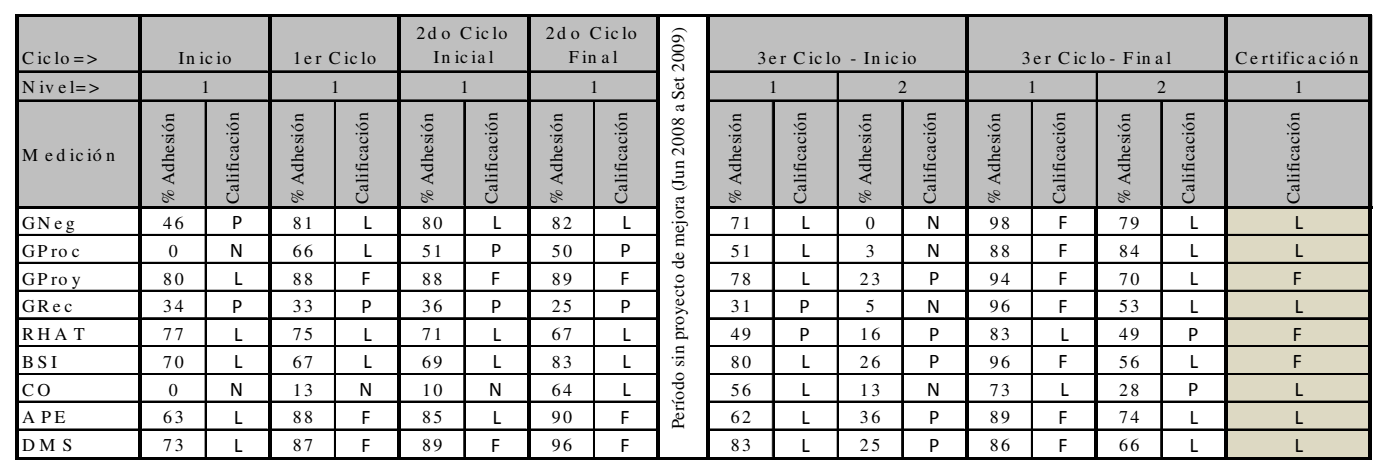

Tabla 1. Adhesión y calificación de procesos de Alfa respecto de MoProSoft

La evaluación inicial se basó en entrevistas al personal de la empresa realizada por el investigador principal y el estudiante, usando un cuestionario a manera de una lista de comprobación (checklist) derivado del modelo MoProSoft y considerando prácticas de evaluación de la ISO/IEC 15504-2 de capacidad de procesos software. Este tipo de evaluación (diagnóstica) tuvo la ventaja de ser rápido (poco tiempo para realizarla) pero como desventaja en que el grado de fiabilidad es menor (de manera 
comparada a otros tipos); sin embargo, para el propósito de las evaluaciones dentro del Proyecto resultó conveniente. Las evaluaciones posteriores se basaron en evidencias y cada vez con mayor rigurosidad para preparar a la organización hacia un proceso de certificación.

De la Tabla 1 se puede apreciar que entre la evaluación inicial y final del primer ciclo (columnas Inicio y 1er ciclo) se incrementa de manera significativa GNeg y GProc, y en menor medida CO, APE y DMS; lo que guarda cierta relación con los objetivos de mejora de dicha iteración: 85\% en los procesos GNeg, GProc y APE. Como los procesos dentro de MoProSoft están relacionados era de esperarse que otros procesos mejoren, como es el caso de DMS y CO; y el resto se mantiene casi en lo mismo. Además se puede apreciar una aparente desmejora en GRec y BSI pero ello es atribuible a la fiabilidad de la herramienta de medición por lo que no es relevante su análisis.

Los resultados de la evaluación inicial en el tercer ciclo denotan cierta desmejora o poco incremento de la capacidad de los procesos. Esto tiene sentido pues Alfa dejó el Proyecto desde Jun-2008 hasta Sep-2009; pero trato de mantener, según sus directivos, los resultados obtenidos en la mejora de procesos. Asimismo, Alfa había decidido seguir creciendo en capacidades de proceso pues ya había obtenido ciertos beneficios comerciales y aspiraba a la certificación de nivel 1 de MoProSoft, por lo que la evaluación inicial y final para el tercer ciclo de mejora consideró el nivel de capacidad 2. Las evaluaciones se realizaron con un nivel medio de rigurosidad lo que es mejor en fiabilidad para que se preparen para la evaluación de certificación. Finalmente, Alfa paso por un proceso riguroso de evaluación con MoProSoft usando un método de evaluación que sí cumplía con la ISO/IEC 15504 con lo que consiguió la certificación.

Dentro de las lecciones aprendidas se pueden señalar las siguientes:

- El compromiso de la alta dirección traducido en la participación y apoyo de toda la organización fue muy importante para lograr los objetivos.

- La sensibilización y continua capacitación facilitó el trabajo de adopción a pesar de algunas dificultades en la operación de Alfa.

- La estrategia de abordar los problemas que mayor impacto tienen en la operación fue muy conveniente pues permitió que el equipo de la empresa progresivamente se interesara por el proyecto al ver que los cambios les facilitaba el trabajo.

- El monitoreo de las mejoras a todo nivel fue muy conveniente hasta que la organización haya adoptado las prácticas como algo natural, pues resultaba fácil que las dejen de lado al menor problema; al ser un tema cultural.

- La documentación de las mejoras (procesos y técnicas) ayudaron a que todos trabajen de la misma manera y puedan comunicarse con menos inconvenientes.

\section{$5 \quad$ Discusión final}

La iniciativa de la PUCP de liderar un proyecto de mejora de procesos (adopción de MoProSoft) en la industria de software conformado en mayor medida por pequeñas empresas ha sido una gran experiencia y una forma interesante de estrechar lazos entre el mundo académico y el mundo empresarial. Por un lado se aprecia que las empresas suelen tener un ritmo agitado tras el día a día buscando cumplir sus objetivos 
comerciales. Por otro lado se aprecia que la Universidad tiene su propia dinámica con tiempos o plazos que suelen ser menos agitados. Sin embargo, fue posible encontrar intereses que pudieron ser manejados por ambos contextos para lograr resultados favorables para ambos involucrados.

Durante la ejecución del proyecto en la Empresa, se pudo comprobar que el marco metodológico que presentó el proyecto COMPETISOFT facilitó la implementación, proporcionando una alternativa para las pymes desarrolladoras de software. Esta experiencia particular, dentro del contexto del Proyecto, contribuye a reforzar el hecho que las capacidades de procesos se han incrementado en la Empresa lo que fue corroborado por la certificación obtenida y que para ello ha sido necesario que se tengan avances progresivos y sostenibles en cada ciclo de mejora.

La experiencia en la industria definitivamente es un componente muy importante pues sirve para reafirmar los aspectos teóricos vistos en clase, para mejorar la forma de transmitir los conocimientos necesarios para los profesionales, para revisar o cuestionar las prácticas mismas de la ingeniería de software aplicado a situaciones particulares como suele ocurrir en proyectos de mejora de procesos.

Los problemas del proceso de mejora en Alfa, fueron muy parecidos a los reportados por las otras empresas del Proyecto [Maidana et al 2008] y los mismos que se han ampliado en su recopilación que se presentarán en otro artículo. En general se puede indicar que los problemas en los pilotos (relativos a actividades y formatos) fueron resueltos por consenso entre los responsables de las actividades y monitoreando los cambios.

La experiencia de los estudiantes (de ese entonces) les ha servido mucho para poder orientar mejor muchas de sus decisiones recientes a nivel profesional. Conocer la empresa completa y cómo ejecutan los procesos les permite tener una idea más clara de cuál es el aporte que pueden realizar ellos mismos en cualquier otra empresa, desempeñando un rol distinto al de responsables de procesos. El nivel de aprendizaje logrado también es muy alto pues cumplió varios roles como capacitador, diseñador de formatos, documentador, planificador y proponente de alternativas, entre otros.

\section{Reconocimientos}

El presente trabajo está enmarcado dentro del proyecto 506AC0287 COMPETISOFT del programa CYTED (1era Fase), DGI-008-2009-PUCP (2da Fase) y FINCYT-009 2010-PIPEA con ACKLIS SAC (3era Fase). Apoyado por el Departamento de Ingeniería de la PUCP.

\section{Referencias}

Allen P. (2003) "PRISMS: an approach to software process improvement for small to medium enterprises". Proceedings of the Third International Conference On Quality Software (QSIC'03). USA (2003)

COMPETISOFT (2008) "Proyecto de mejora de procesos para fomentar la competitividad de la pequeña y mediana industria de software de Iberoamérica", http://alarcos.inf-cr.uclm.es/Competisoft/ [Revisado 2008-08-15]. 
Gresse, C., Anacleto, A., Salviano, C. (2006) "Helping Small Companies Assess Software Processes", IEEE Software, Jan-Feb 2006, pp. 91-98.

Hurtado, J., Bastarrica, C. (2005) "Hacia una Línea de Procesos Ágiles Agile SPsL". http://www.dcc.uchile.cl/ cecilia/papers/AgileSPsL.pdf [Revisado 2007-09-15].

ISO (2003) "ISO/IEC 15504-2:2003 Information Technology - Process assessment Part 2 Performing an assessment". Geneva.

ISO (2011) "ISO/IEC 29110-5-1-2:2011 Software engineering — Lifecycle profiles for Very Small Entities (VSEs) - Part 5-1-2: Management and engineering guide: Generic profile group: Basic profile", Geneva, January.

Jones J. (2005), "Process Improvement in a Small Company". Proceedings of the First International Research Workshop for Process Improvement in Small Settings, 2005 Special Reports CMU/SEI-2006-SR001, 06sr001, pp. 251-265.

Laporte, C. Simon, A. Renault, A. (2008), "The application of International Software engineering Standards in Very Small enterprise" in SQP Vol. 10, $\mathrm{N}^{\circ} 3$, pp 4-11. ASQ.

NTP (2009) "NTP 291.100 Ingeniería de Software. Modelos de procesos y evaluación para desarrollo y mantenimiento de software. Parte 1: Definición de conceptos y productos". Lima.

Maidana, E., Vilchez, N., Vega, J., Dávila, A. (2008). "Identificación de problemas en proyectos de mejora de procesos: una experiencia en tres pequeñas empresas desarrolladoras de software en el Perú". Proc. VII Jornada Peruana de Computación. 120-129.

Oktaba, H., Alquicira, C., Su, A., Palacios, J., Pérez, C., López, F. (2004) "Método de Evaluación de procesos para la industria del software EvalProSoft", V 1.1. México.

Oktaba, H., Alquicira, C., Su, A., Martínez, A., Quintanilla, G., Ruvalcaba, M., López, F., Rivera, M., Orozco, M., Fernández, Y., Flores, M. (2005) "Modelo de Procesos para la Industria del Software MoProSoft”. Versión 1.3, México.

Oktaba, H. (2006) "MoProSoft: A software process model for small Enterprises". Proceeding of the First International Research Workshop for Process Improvement in Small Setting. Special Reports CMU/SEI-2006-SR001, 06sr001, pp 93-101.

Oktaba, H., Alquicira, C., Piattini M., García F., Ruiz F., Pino F., (2007). "Software Process Improvement: The Competisoft Project". IEEE-Computer. Vol. 6, No 2, pp. 21-28.

Pino, F., García, F., Ruíz, F., Piattini, M. (2006). “Adaptación de las normas ISO/IEC 12207:2002 y ISO/IEC 15504:2003 para la evaluación de la madurez de procesos software en países en desarrollo", IEEE América Latina, Vol. 4, № 2, pp. 17-24.

Richardson, I., Gresse, C. (2007). "Why are small software organizations different?" IEEE Software. Vol.24, No.1, Jan-Feb 2007, pp. 18 - 22.

Weber K. Rocha A. (2004) "Modelo de Referência para Melhoria de Processo de Software: uma abordagem brasileira". Proc. of the QUATIC 2004, 73-78. 